\title{
Polyphase alternating codes
}

\author{
M. Markkanen ${ }^{1}$, J. Vierinen ${ }^{2}$, and J. Markkanen ${ }^{3}$ \\ ${ }^{1}$ Eigenor Corporation, Lompolontie 1, 99600 Sodanykylä, Finland \\ ${ }^{2}$ Sodankylä Geophysical Obervatory, Tähteläntie 62, 99600 Sodankylä, Finland \\ ${ }^{3}$ EISCAT Scientific Association, Tähteläntie 54B, 99600 Sodankylä, Finland
}

Received: 29 November 2007 - Revised: 29 February 2008 - Accepted: 15 April 2008 - Published: 5 August 2008

\begin{abstract}
We present a new class of alternating codes. Instead of the customary binary phase codes, the new codes utilize either $p$ or $p-1$ phases, where $p$ is a prime number. The first class of codes has code length $p^{m}$, where $m$ is a positive integer, the second class has code length $p-1$. We give an actual construction algorithm, and explain the principles behind it. We handle a few specific examples in detail. The new codes offer an enlarged collection of code lengths for radar experiments.
\end{abstract}

Keywords. Radio science (Ionospheric physics; Instruments and techniques)

\section{Introduction}

Alternating codes are widely used in incoherent scatter radar measurements and their properties are well known. There are two classes of these codes. The so called type 1 codes were introduced by Lehtinen (1986). The number of bauds in any single transmission of a type 1 code, i.e. the code length, is a power of two. Also the number of transmissions, i.e. the code set size, is a power of two, which sometimes causes inflexibility when designing radar experiments. With this in mind, Sulzer (1993) proposed a new type of alternating codes (type 2) which made it possible to use other code lengths. These codes also produce unambiguous back-scatter autocorrelation function estimates of the target, but there are no efficient search strategies for finding longer type 2 codes. The longest type 2 alternating code that we know about has length $14^{1}$.

\section{Correspondence to: J. Vierinen}

(juha.vierinen@iki.fi)

\footnotetext{
${ }^{1}$ The 8 sequences forming the 14-bit code are in hexadecimal format: 406, b72, 4df, bab, 24c, d38, 295 and de1.
}

In this paper we generalize previous work (Markkanen and Nygrén, 1997) and apply it to polyphase codes, i.e. codes that are phase coded with two or more different phases. We have identified two different classes of polyphase alternating codes. The first class includes codes that have $p$ phases and code lengths $p^{m}$, where $p$ is a prime number and $m$ is a positive integer. The second class contains codes with $p-$ 1 phases and code length $p-1$. The number of codes in a code set of either class is equal to the (common) length of the codes belonging to the set. The new codes increase the number of available code lengths considerably, as shown in Fig. 1. Within codes shorter than 130, there are eight lengths of binary codes and additional 64 lengths of polyphase codes. Often there are several alternating codes of a given length, so that there are altogether 763 essentially different code sets shorter than 130 .

This paper concentrates on polyphase alternating codes satisfying the so called weak condition. Strong codes can be generated from weak codes using the method described by Sulzer (1989), which also works for the polyphase alternating codes presented here. To create an alternating code set satisfying the strong condition, the code set is duplicated, and in each code in the duplicated part, bauds with even indices are multiplied by -1 .

The key property of the new codes (of the length $p^{m}$ ) is a close similarity between individual codes in a code set: apart from one code, the codes are essentially cyclic permutations of each other. In the next sections we show that an alternating code set having the cyclic property can be constructed if, and only if, the number of phases in the code is a prime number. The role of cyclic permutations was originally found while searching regularities in binary alternating codes, but their role in constructing polyphase alternating codes for primes larger than 2 was not realized at that time. 

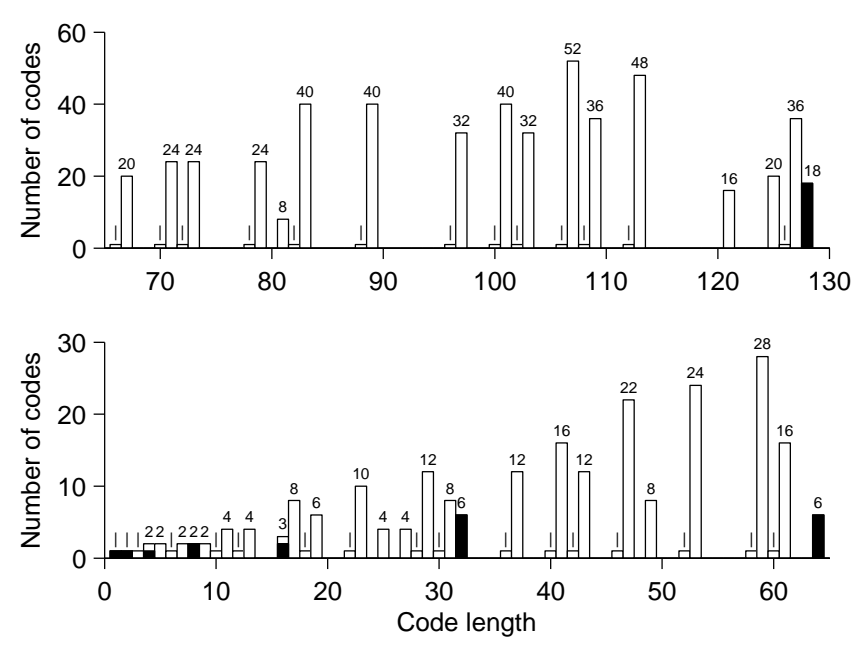

Fig. 1. Number of different alternating codes up to code length 130. The black bars represent the well-known binary phase alternating codes, the white bars represent new polyphase alternating codes.

\section{Construction of $p$-nary alternating codes}

By a $p$-nary sequence ( $p$-nary pulse) we mean a phase modulated pulse where the complex phase factors of the bauds belong to the set $E_{p}=\left\{\alpha_{i}=e^{i 2 \pi \sqrt{-1} / p} \mid i=0, \ldots, p-1\right\}$. In what follows, $p$ is a prime number unless otherwise stated. A $p$ nary alternating code of length $n+1$ and size $n+1$ is a set $\left\{A_{k}=\left(a_{k, 0}, \ldots, a_{k, n}\right) \mid k=0, \ldots, n\right\}$ of $p$-nary pulses, which satisfies the natural extension of the weak condition given in Lehtinen (1986) for binary codes:

Condition 1. For each $i, i^{\prime}, j$ and $j^{\prime}$ where $j-i=j^{\prime}-i^{\prime}, i \neq j$, and $i \neq i^{\prime}$

$\sum_{k=0}^{n} a_{k, i} \overline{a_{k, j}} \overline{a_{k, i^{\prime}} \overline{a_{k, j^{\prime}}}}=0$.

We now first consider codes of length $n$, and later increase the length to $n+1$. We denote by $M$ the operator which shifts the elements of a sequence cyclically by one, that is

$M\left(a_{0}, \ldots, a_{n-1}\right)=\left(a_{1}, \ldots, a_{n-1}, a_{0}\right)$.

Let $A=\left(a_{0}, \ldots, a_{n-1}\right)$ be a $p$-nary sequence and let us denote by $U$ the unit sequence $U=(1,1, \ldots, 1)$. By multiplication of two sequences we mean pointwise multiplication $\otimes$, and by the conjugate of $A$ we mean pointwise complex conjugate $\bar{A}$. Then $A \otimes \bar{A}=U$, because $\left|a_{i}\right|=1$ for all $i$.

Let $C_{A}$ be the set of sequences generated by the pulse $A$, $C_{A}=\left\{A, M A, \ldots, M^{n-1} A, U\right\}$, where $M^{i}$ means $i$ repeated cyclical shift operations. We will show that $C_{A}$ constitutes a $p$-nary alternating code set, if the following two conditions are met.

Condition 2a. All the sequences in $C_{A}$ are different.
Condition 3a. Multiplying all the sequences of $C_{A}$ by a fixed sequence in $C_{A}$ permutes $C_{A}$.

To prove that these conditions are sufficient, we need the following properties 1 and 2 of the sequences satisfying conditions $2 \mathrm{a}$ and $3 \mathrm{a}$.

Property 1. The sum s of elements of $A$ is -1 .

This can be seen by considering the sequence $S=A+M A+\cdots+M^{n-1} A+U$, which equals $(s+1) U$ (the sequences $A, \ldots, M^{n-1} A$ contain in each location all the elements of $A$ in some cyclic order). Because by condition 3 a multiplication by $A$ permutes the terms of $S$, $A \otimes S=S$, and because $A \otimes U=A$, it follows that

$(s+1) A=(s+1) U$.

Now condition 2 a requires that $A \neq U$. Thus $s+1=0$ and $s=-1$. Notice that in the case of $p=2$, when $E_{p}=\{1,-1\}$, it follows from property 1 that $n$ is odd.

Property 2. There exists an $i_{0}$ such that $\overline{M^{i} A}=M^{i+i_{0}} A$ for all $i$.

By condition 3a there is an $i_{0}$ such that $A \otimes M^{i_{0}} A=U$, that is, $M^{i_{0}} A=\bar{A}$. Then

$\overline{M^{i} A}=M^{i} \bar{A}=M^{i} M^{i_{0}} A=M^{i+i_{0}} A, \quad$ for any $i$.

We can now show that the condition 1 holds. We denote by $A^{i}$ the sequence formed by taking the $i$ th elements of the sequences $A_{0}, \ldots, A_{n-1}$. Condition 1 is then the requirement that the sum of elements of sequence $D=A^{i} \otimes \overline{A^{j}} \otimes \overline{A^{i^{\prime}} \otimes \overline{A^{j^{\prime}}}}$, added by 1 (coming from $A_{n}=U$ ), is 0 . That is, the sum of elements of $D$ is -1 .

Now $A^{i}=M^{i} A$ and thus

$D=M^{i} A \otimes \overline{M^{j} A} \otimes \overline{M^{i^{\prime}} A \otimes \overline{M^{j^{\prime}} A}}$.

Property 2 says that $D \in C$ and if we can show that $D \neq U$, it follows from property 1 that the sum of elements of $D$ is indeed -1 .

Because $B_{1} \otimes \overline{B_{2}} \neq U$ for any $B_{1} \neq B_{2}$ when elements of $B_{1}$ and $B_{2}$ have absolute value 1 , it is enough to show that

$M^{i} A \otimes \overline{M^{j} A} \neq M^{i^{\prime}} A \otimes \overline{M^{j^{\prime}} A}$.

The left hand side is $M^{i} A \otimes M^{j+i_{0}} A=M^{i}\left(A \otimes M^{j-i+i_{0}} A\right)$ and similarly the right hand side is $M^{i^{\prime}}\left(A \otimes M^{j^{\prime}-i^{\prime}+i_{0}} A\right)$. Because $j-i=j^{\prime}-i^{\prime}$, the sequences inside parentheses are equal, and because $i \neq j$, they are not equal to $U$. Then indeed $M^{i}(\ldots) \neq M^{i^{\prime}}(\ldots)$, because $i \neq i^{\prime}$.

Finally, we will make the number of codes and the their length equal by copying the first element of each sequence to the end of the sequence. Then $A^{n}=A=M^{n} A$. To see that condition 1 is still satisfied, let us first suppose that $n$ is in the set $\left\{i, j, i^{\prime}, j^{\prime}\right\}$ but 0 is not. Then condition 1 follows 
trivially from what was said above (by dropping the first element of each sequence we have the same codes, only in order $\left.M A, \ldots, M^{n-1} A, A, U\right)$. If both 0 and $n$ belong to the set $\left\{i, j, i^{\prime}, j^{\prime}\right\}$, the only possibility is $i=0, j=i^{\prime}=n / 2$. Then $n$ must be even, so we can suppose that $p \neq 2$. With the above choice of indices the left hand side of Eq. (1) is $A \otimes \overline{M^{n / 2} A}$ and the right hand side is $M^{n / 2} A \otimes \bar{A}$. As they are conjugates, they are unequal, unless they are both equal to $U$. But $M^{n / 2} A \neq A$ and thus $A \otimes \overline{M^{n / 2} A} \neq A \otimes \bar{A}=U$, and so $D \neq U$, meaning that condition 1 is satisfied also in this case.

The requirement that $p$ is prime is necessary for this method of constructing alternating codes. With composite $p$ there isn't any sequences $A$ satisfying conditions $2 \mathrm{a}$ and $3 \mathrm{a}$. The essential reason is that for composite $p$ the sequences $A, A \otimes A, A \otimes A \otimes A, \ldots$ can have different number of ones.

\section{Construction of the sequence $A$}

For the construction of sequences $A$ generating alternating codes, it will be advantageous, instead of $E_{p}$ to consider the set of possible exponents of $\alpha$, the integers $\mathbb{Z}_{p}=\{0, \ldots, p-1\}$ with $\bmod p$-arithmetic. The sequences with elements in $E_{p}$ correspond to vectors in the vector space $\mathbb{Z}_{p}^{n}=\mathbb{Z}_{p} \times \ldots \times \mathbb{Z}_{p}$, multiplication of sequences corresponds to addition of vectors and the unit sequence $U$ corresponds to the null vector $\mathbf{0} \in \mathbb{Z}_{p}^{n}$. The operator $M$ corresponds to the linear operator mapping each base vector of $\mathbb{Z}_{p}^{n}$ cyclically to the previous one.

We now have a vector $A=\left(a_{0}, \ldots, a_{n-1}\right) \in \mathbb{Z}_{p}^{n}$ and the set $C_{A}=\left\{A, M A, \ldots, M^{n-1} A, \mathbf{0}\right\} \subset \mathbb{Z}_{p}^{n}$. Conditions $2 \mathrm{a}$ and $3 \mathrm{a}$ correspond to the following conditions.

Condition 2b. All the vectors in $C_{A}$ are different.

Condition 3b. Adding a fixed vector of $C_{A}$ to all vectors belonging to $C_{A}$, permutes $C_{A}$.

The sum of any two vectors in $C_{A}$ belong to $C_{A}$ by condition 3b. Because for any $B \in C_{A}$ also $2 B=B+B \in C_{A}$ and similarly for any $k \in \mathbb{Z}_{p}$, it follows that $C_{A}$ is a vector subspace of $\mathbb{Z}_{p}^{n}$. With $m$ denoting the dimension of $C_{A}$, the number of vectors in $C_{A}$ is $p^{m}$, and thus $n=p^{m}-1$.

Because $M C_{A}=C_{A}, C_{A}$ is an $M$-invariant subspace, and one could use the theory of invariant subspaces to show the existence of alternating codes, and to construct the codes. However, here we present a more elementary derivation of the construction algorithm.

Condition $3 \mathrm{~b}$ means that for most indices $j$, there is an index $k$ such that $A_{k}=A+A_{j}$, implying that $a_{0}, a_{1}, \ldots$ satisfy for all $i$ linear relations $a_{k+i}=a_{i}+a_{j+i}$. As solutions of linear difference equations satisfy these kind of relations, it seems plausible to try to find the sequence $A$ as a solution of a suitably chosen difference equation.
It turns out that the proper order for the difference equation is $m$, so let us consider in $\mathbb{Z}_{p}$ a difference equation

$x_{i+m}=b_{m-1} x_{i+m-1}+\ldots+b_{0} x_{i}, \quad i=0, \ldots$,

with $b_{0}, \ldots, b_{m-1} \in \mathbb{Z}_{p}$. Because (a) any $m$-tuple of consecutive elements of the solution $\left(x_{i}\right)$ fixes all subsequent elements of the solution, and (b) there are only finite number (at most $p^{m}$ ) of such $m$-tuples, the solution is essentially periodic (the sequence can start with a non-periodic part).

Let us suppose that Eq. (2) has a solution $A^{\prime}=\left\{a_{i}\right\}_{i=0}^{\infty}$ with period $n=p^{m}-1$. We will now show that the vector $A=\left(a_{0}, \ldots, a_{n-1}\right) \in \mathbb{Z}_{p}^{n}$ satisfies conditions $2 \mathrm{~b}$ and $3 \mathrm{~b}$. The periodic part of $A^{\prime}$ contains all the $p^{m}-1$ different non-zero $m$-tuples, and so $A^{\prime}$ can not have a non-periodic start. Then $a_{n}=a_{0}, a_{n+1}=a_{1}, \ldots$, and the $m$-tuples $\left(a_{0}, \ldots, a_{m-1}\right)$, $\left(a_{1}, \ldots, a_{m}\right), \ldots,\left(a_{n-1}, \ldots, a_{n+m-2}\right),(0, \ldots, 0)$, which are the starts of vectors $A, M A, \ldots, M^{n-1} A, \mathbf{0}$, are all the $p^{m}$ different $m$-tuples of $\mathbb{Z}_{p}$. This proves condition $2 \mathrm{~b}$. If $B_{1}, B_{2} \in C_{A}$, there is $B_{3} \in C_{A}$ such that the first $m$ elements of $B_{1}+B_{2}$ are equal to the first $m$ elements of $B_{3}$. Because $B_{1}+B_{2}$ is also a solution of Eq. (2), it follows that $B_{1}+B_{2}=B_{3}$. This proves condition $3 \mathrm{~b}$.

Notice that we can choose as our $A$ any nonzero vector of $\mathbb{Z}_{p}^{n}$, e.g. one starting with $m-1$ zeros followed by 1 .

We can summarize the preceding discussion by the following result.

Theorem. If a $m$ th-order difference equation in $\mathbb{Z}_{p}$ is such that it has a solution with period $p^{m}-1$, the first $p^{m}-1$ elements of the solution can be used as a generator of a p-nary alternating code.

\section{The number of $p^{m}$-type alternating codes}

If all the roots of polynomial $Q(x)=x^{m}-b_{m-1} x^{m-1}-\ldots-b_{0}$ are different, the general solution of the difference Eq. (2) is

$x_{i}=c_{1} \alpha_{1}^{i}+\ldots+c_{m} \alpha_{m}^{i}, \quad i=0, \ldots$,

where $\alpha_{1}, \ldots, \alpha_{m}$ are the roots of $Q(x)$ and $c_{1}, \ldots, c_{m}$ are arbitrary coefficients. If (A) $Q(x)$ is irreducible (in $\mathbb{Z}_{p}[x]$ ) and (B) there is no integer $d$ smaller than $n=p^{m}-1$ such that $Q(x)$ divides $x^{d}-1$, then the roots of $Q(x)$ are different and all the sequences $\left(1, \alpha_{i}, \alpha_{i}^{2}, \ldots\right)$ have common period $n$. Then also the period of the sequence $\left(x_{i}\right)$ is $n$. Thus each $m$ th-degree polynomial $Q(x) \in \mathbb{Z}_{p}[x]$ that satisfies (A) and $(\mathrm{B})$, determines a $p$-nary alternating code.

One can see that different polynomials $Q$ determine different codes by noting that an element in the solution sequence following an $m$-tuple of form $(0, \ldots, 0,1,0, \ldots, 0)$, with the 1 in the $k$ th place, is the corresponding coefficient $b_{k}$ of $Q$. 
It is possible to show (e.g. Lidl and Niederreiter, 1997, p. 85) that the number of different $m$ th-degree polynomials $N_{c}$ satisfying the conditions (A) and (B) is

$N_{c}=\frac{\varphi\left(p^{m}-1\right)}{m}$.

Here $\varphi(i)$ is the Euler $\varphi$-function, which is the number of integers smaller than $i$ which do not have a common factor with $i$. It can also be shown that there are no other sets $C$ satisfying conditions $2 \mathrm{~b}$ and $3 \mathrm{~b}$, so the number $N_{c}$ is the number of different alternating codes satisfying those conditions. This means especially that for any prime number $p$ and any positive integer $m$ there exist $p$-nary alternating codes of length $p^{m}$.

\section{$5 \quad p$-nary alternating codes of length $p$}

We will now look more closely at the case $m=1$. In this case the difference equation is simply

$x_{i+1}=b_{0} x_{i} \quad b_{0} \in \mathbb{Z}_{p}, \quad i=0,1, \ldots$,

and if we choose $x_{0}=1$, its solution is $x_{i}=b_{0}^{i}(\bmod p)$. We get thus a suitable $A$ if and only if all the numbers $1, b_{0}, \ldots, b_{0}^{p-2} \bmod p$ are different, that is, if $b_{0}$ is the generator of the cyclic multiplicative group $\mathbb{Z}_{p}^{*}$.

As an example, the only generator of $\mathbb{Z}_{3}^{*}$ is $b_{0}=2$, giving the set of sequences

$C=\{(1,2,1),(2,1,2),(0,0,0)\}$

of exponents of the basic phase factor $\alpha=e^{2 \pi \sqrt{-1} / 3}$ of the 3 -nary code. For $\mathbb{Z}_{5}^{*}$ there are two generators $b_{0}=2$ and 3 , giving the sets of exponents

$$
\begin{aligned}
C=\{ & (1,2,4,3,1),(2,4,3,1,2),(4,3,1,2,4), \\
& (3,1,2,4,3),(0,0,0,0,0)\}
\end{aligned}
$$

and

$$
\begin{aligned}
C=\{ & (1,3,4,2,1),(3,4,2,1,3),(4,2,1,3,4), \\
& (2,1,3,4,2),(0,0,0,0,0)\}
\end{aligned}
$$

for $\alpha=e^{2 \pi \sqrt{-1} / 5}$.

Let $b_{0}$ be a generator of $\mathbb{Z}_{p}^{*}$ and $A$ the solution of the corresponding difference Eq. (3). It was shown in Sect. 3 that the sequence $k A$ belongs to the code for all $k=0, \ldots, p-1$, and we can change the order of sequences to have $C=\{\mathbf{0}, A, 2 A, \ldots,(p-1) A\}$. This means that the columns of the alternating code are suitably chosen columns of the Fourier matrix $F_{p}=\left(f_{i j}\right)$ with $f_{i j}=e^{i j 2 \pi \sqrt{-1} / p}$.

The columns of $F_{p}$ are orthogonal and form a closed set under pointwise multiplication. This is true for arbitrary (also non-prime) $p$ and we can use the columns, or rather, the columns consisting of the corresponding exponents, for searching $p$-nary alternating codes for any $p$. Using the $F_{p}$ is analogous to the use of Walsh sequences in Lehtinen (1986).
Because all the pointwise products of columns of $F_{p}$ and their conjugates are also columns of $F_{p}$, a set $C$ is an alternating code if the pointwise products of those columns that correspond to the indices of condition 1 are different from $U$. Multiplication by complex conjugate corresponds to subtraction of exponents, and so for arbitrary $p$ the set $C_{A}=\{\mathbf{0}, A, 2 A, \ldots,(p-1) A\}$ with the generating sequence $A=\left(a_{0}, \ldots, a_{n-1}\right) \in \mathbb{Z}_{p}^{n}$ is an alternating code if $A$ satisfies the following condition (analogous to the condition for Walsh indices given in Lehtinen (1986)).

Condition 4. For each $i, i^{\prime}, j$ and $j^{\prime}$ where $j-i=j^{\prime}-i^{\prime}, i \neq j$, and $i \neq i^{\prime}$,

$\left(a_{i}-a_{j}\right)-\left(a_{i^{\prime}}-a_{j^{\prime}}\right) \neq 0$.

The condition 4 can be rephrased: all differences (mod $p$ ) of values $a_{i}, a_{j}$ of elements of $A$ with fixed difference of indices $i, j$ are different.

We will now consider again for a prime $p$ the sequence $A=\left(1, b_{0}, \ldots, b_{0}^{p-2}\right) \bmod p$, which generates an alternating code of length $p-1$ (we drop the duplicate element from the end).

Because of periodicity of $b_{0}^{i}$ with respect to $i$ the sequence $A$ satisfies an even stronger condition: all differences ( mod $p$ ) of values $a_{i}, a_{j}$ of elements of $A_{1}$ with fixed difference $(\bmod p-1)$ of indices $i, j$ are different. Then it trivially satisfies also the following condition: all differences $(\bmod p-1)$ of indices $i, j$ of elements of $A$ with fixed difference $(\bmod p)$ of values $a_{i}, a_{j}$ are different.

For the "dual" sequence $A^{\prime}$ with indices $1, b_{0}, \ldots, b_{0}^{p-2}$ and corresponding values $0,1 \ldots, p-2$ this means that all differences $(\bmod p-1)$ of values of elements of $A^{\prime}$ with fixed difference $(\bmod p)$ of indices are different. But this is again stronger than the condition for $(p-1)$-nary alternating codes: all differences $(\bmod p-1)$ of values of elements of $A^{\prime}$ with fixed difference of indices are different. Thus the sequence $A^{\prime}$ generates a ( $\left.p-1\right)$-nary alternating code of length $p-1$.

Example. Dual 7-nary and 6-nary sequences. When $p=7$ and $b_{0}=3$, we have the following situation:

$\begin{array}{cccccccc}\mathbb{Z}_{7} & & & & & & & \mathbb{Z}_{6} \\ \text { index: } & 0 & 1 & 2 & 3 & 4 & 5 & \text { :value } \\ \text { value: } & 1 & 3 & 2 & 6 & 4 & 5 & \text { :index }\end{array}$

This gives the sequence $(1,3,2,6,4,5,1)$ for generating a 7 -nary alternating code and the sequence $(0,2,1,4,5,3)$ for generating a 6-nary alternating code.

It can be noted that whereas in $p$-nary codes the first and last columns are identical and the constant column of $F_{p}$ is not in the code, in $(p-1)$-nary codes all the columns of $F_{p-1}$ are in the code exactly once.

Due to the close connection between the $p$-nary and ( $p-$ 1)-nary alternating codes, one might think that the number of 
$p$-nary and ( $p-1)$-nary alternating codes is the same. However, this is not the case. Different $p$-nary codes correspond to different sequences of the same ( $p-1)$-nary code, and thus the construction presented here gives only one $(p-1)$-nary alternating code for each prime $p$. This can be seen by noticing that if $b_{0}$ and $b_{1}$ are generators of $\mathbb{Z}_{p}^{*}$, there is a $k_{0}$ such that $b_{0}=b_{1}^{k_{0}}$. If then $A=\left\{a_{1}, a_{2}, \ldots\right\}$ and $A^{\prime}=\left\{a_{1}^{\prime}, a_{2}^{\prime}, \ldots\right\}$ are dual sequences of $\left\{1, b_{0}, \ldots\right\}$ and $\left\{1, b_{1}, \ldots\right\}$,

$a_{\left(b_{0}^{k}\right)}^{\prime}=a_{\left(b_{1}^{k_{0}}\right)}^{\prime}=k_{0} k=k_{0} a_{\left(b_{0}^{k}\right)}$,

implying that $A^{\prime}=k_{0} A$.

\section{Algorithm for generating $p^{m}$-length alternating codes}

Finding alternating codes is a fairly simple computation which involves going through all the $p^{m}$ different $m$-tuples $b=\left(b_{0}, \ldots, b_{m-1}\right)$ with elements in $\mathbb{Z}_{p}$.

For each $b$, we generate a solution of the corresponding $m$ th order difference equation (2) starting with the the initial values $x_{i}=0$ for $i=0 \ldots m-2$ and $i_{m-1}=1$ (notice that the solution is calculated in modulo $p$ arithmetic). Next we check if the solution $\left(x_{i}\right)$ has period $p^{m}-1$. If it has, then this solution can be used to generate an alternating code set. The resulting code is

$$
\begin{aligned}
A_{j} & =\left(\alpha^{x_{j}}, \ldots, \alpha^{x_{j+p^{m}-1}}\right), \quad j=0, \ldots, p^{m}-2, \\
A_{p^{m}-1} & =(1,1, \ldots, 1),
\end{aligned}
$$

where $\alpha=e^{2 \pi \sqrt{-1} / p}$.

To generate a ( $p-1)$-nary code, one first constructs the generator of the corresponding $p$-nary code and then forms the $(p-1)$-nary code set using the transformation described in Sect. 5.

Table 1 lists alternating codes for the first few hundred code lengths, one code per code length. The codes are expressed in terms of number of phases $p$, generating coefficients $b=\left(b_{0}, \ldots, b_{m-1}\right)$ and code length. As an example, Fig. 2 shows a 25 baud alternating code set. A program for generating weak and strong alternating codes is available at: http://mep.fi/ac, the program is also available as an online supplement at http://www.ann-geophys.net/26/2237/ 2008/angeo-26-2237-2008-supplement.zip.

\section{Discussion}

It is also possible to use truncated polyphase alternating codes in a similar manner as binary phase alternating codes, in order to have smaller number of bauds.

It has been shown in Lehtinen et al. (1997) that the nonrandomized binary strong alternating codes have as bad a covariance behaviour as possible (in the sense explained there). That behaviour was shown to depend only on the conditions that for the corresponding weak code the sum of elements of

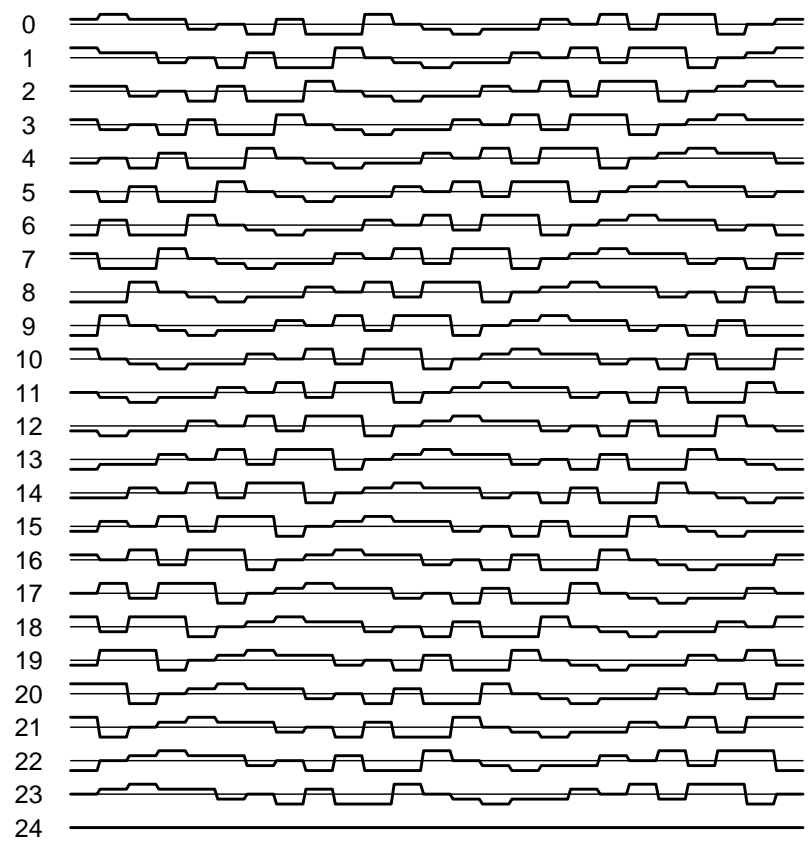

Fig. 2. The phases of a 25 baud 5-nary alternating code. The cyclic nature of the code set is evident. One can also see property 2, which implies that each code is conjugate symmetric.

any column $A^{i}$ in the matrix that has the code sequences $A_{k}$ as rows, is 0 , and that for any $i, j$, the product $A^{i} \otimes A^{j}$ of two columns is either $U$ or $A^{k}$ for some $k$. As all the polyphase alternating codes presented in this paper also satisfy those conditions, the corresponding strong codes have bad covariance behaviour and will benefit from randomization. We note that it is possible to randomize any alternating code set with arbitrary phase factors.

As arbitrary waveform generators have become more common in radar signal processing hardware, transmission of complicated codes such as the polyphase codes presented in this paper have become practical. Because these codes also have constant amplitude, there is no transmission power trade-off compared to binary phase codes. Given the fact that polyphase alternating codes have the same properties as binary phase alternating codes, it should be relatively simple to modify existing correlators and analysis programs to use these new codes.

One benefit of polyphase alternating codes is the larger set of code lengths compared to binary phase codes. This helps in radar experiment design where one wants to have as many bauds as possible (for good spacial resolution) but at the same time wants to keep the code cycle is short as possible (because of rapidly changing targets). With increasing computing power, handling longer codes, (say) up to 128 bauds is possible. The new codes may help in optimizing experiment parameters especially for these longer codes. 
Table 1. Alternating codes with lengths up to 366. The number of phases is denoted by $N_{p}$, the number of different code sets $N_{c}$ and the generator coefficients are denoted by $b$. Only one generator $b$ is given for each code length. The $(p-1)$-nary codes are generated from the next consecutive prime.

\begin{tabular}{|c|c|c|c|c|c|c|c|c|c|c|c|}
\hline Length & $N_{p}$ & $N_{c}$ & $b$ & Length & $N_{p}$ & $N_{c}$ & $b$ & Length & $N_{p}$ & $N_{c}$ & $b$ \\
\hline 2 & 2 & 1 & 1 & 88 & 88 & 1 & $\downarrow$ & 226 & 226 & 1 & $\downarrow$ \\
\hline 3 & 3 & 1 & 2 & 89 & 89 & 40 & 3 & 227 & 227 & 112 & 2 \\
\hline 4 & 2 & 1 & 1,1 & 96 & 96 & 1 & $\downarrow$ & 228 & 228 & 1 & $\downarrow$ \\
\hline 4 & 4 & 1 & $\downarrow$ & 97 & 97 & 32 & 5 & 229 & 229 & 72 & 6 \\
\hline 5 & 5 & 2 & 2 & 100 & 100 & 1 & $\downarrow$ & 232 & 232 & 1 & $\downarrow$ \\
\hline 6 & 6 & 1 & $\downarrow$ & 101 & 101 & 40 & 2 & 233 & 233 & 112 & 3 \\
\hline 7 & 7 & 2 & 3 & 102 & 102 & 1 & $\downarrow$ & 238 & 238 & 1 & $\downarrow$ \\
\hline 8 & 2 & 2 & $1,0,1$ & 103 & 103 & 32 & 5 & 239 & 239 & 96 & 7 \\
\hline 9 & 3 & 2 & 1,1 & 106 & 106 & 1 & $\downarrow$ & 240 & 240 & 1 & $\downarrow$ \\
\hline 10 & 10 & 1 & $\downarrow$ & 107 & 107 & 52 & 2 & 241 & 241 & 64 & 7 \\
\hline 11 & 11 & 4 & 2 & 108 & 108 & 1 & $\downarrow$ & 243 & 3 & 22 & $2,0,0,0,1$ \\
\hline 12 & 12 & 1 & $\downarrow$ & 109 & 109 & 36 & 6 & 250 & 250 & 1 & $\downarrow$ \\
\hline 13 & 13 & 4 & 2 & 112 & 112 & 1 & $\downarrow$ & 251 & 251 & 100 & 6 \\
\hline 16 & 2 & 2 & $1,0,0,1$ & 113 & 113 & 48 & 3 & 256 & 2 & 16 & $1,0,0,0,1,1,1,0$ \\
\hline 16 & 16 & 1 & $\downarrow$ & 121 & 11 & 16 & 3,1 & 256 & 256 & 1 & $\downarrow$ \\
\hline 17 & 17 & 8 & 3 & 125 & 5 & 20 & $2,0,1$ & 257 & 257 & 128 & 3 \\
\hline 18 & 18 & 1 & $\downarrow$ & 126 & 126 & 1 & $\downarrow$ & 262 & 262 & 1 & $\downarrow$ \\
\hline 19 & 19 & 6 & 2 & 127 & 127 & 36 & 3 & 263 & 263 & 130 & 5 \\
\hline 22 & 22 & 1 & $\downarrow$ & 128 & 2 & 18 & $1,0,0,0,0,0,1$ & 268 & 268 & 1 & $\downarrow$ \\
\hline 23 & 23 & 10 & 5 & 130 & 130 & 1 & $\downarrow$ & 269 & 269 & 132 & 2 \\
\hline 25 & 5 & 4 & 2,2 & 131 & 131 & 48 & 2 & 270 & 270 & 1 & $\downarrow$ \\
\hline 27 & 3 & 4 & $2,0,1$ & 136 & 136 & 1 & $\downarrow$ & 271 & 271 & 72 & 6 \\
\hline 28 & 28 & 1 & $\downarrow$ & 137 & 137 & 64 & 3 & 276 & 276 & 1 & $\downarrow$ \\
\hline 29 & 29 & 12 & 2 & 138 & 138 & 1 & $\downarrow$ & 277 & 277 & 88 & 5 \\
\hline 30 & 30 & 1 & $\downarrow$ & 139 & 139 & 44 & 2 & 280 & 280 & 1 & $\downarrow$ \\
\hline 31 & 31 & 8 & 3 & 148 & 148 & 1 & $\downarrow$ & 281 & 281 & 96 & 3 \\
\hline 32 & 2 & 6 & $1,0,0,1,0$ & 149 & 149 & 72 & 2 & 282 & 282 & 1 & $\downarrow$ \\
\hline 36 & 36 & 1 & $\downarrow$ & 150 & 150 & 1 & $\downarrow$ & 283 & 283 & 92 & 3 \\
\hline 37 & 37 & 12 & 2 & 151 & 151 & 40 & 6 & 289 & 17 & 48 & 3,4 \\
\hline 40 & 40 & 1 & $\downarrow$ & 156 & 156 & 1 & $\downarrow$ & 292 & 292 & 1 & $\downarrow$ \\
\hline 41 & 41 & 16 & 6 & 157 & 157 & 48 & 5 & 293 & 293 & 144 & 2 \\
\hline 42 & 42 & 1 & $\downarrow$ & 162 & 162 & 1 & $\downarrow$ & 306 & 306 & 1 & $\downarrow$ \\
\hline 43 & 43 & 12 & 3 & 163 & 163 & 54 & 2 & 307 & 307 & 96 & 5 \\
\hline 46 & 46 & 1 & $\downarrow$ & 166 & 166 & 1 & $\downarrow$ & 310 & 310 & 1 & $\downarrow$ \\
\hline 47 & 47 & 22 & 5 & 167 & 167 & 82 & 5 & 311 & 311 & 120 & 17 \\
\hline 49 & 7 & 8 & 2,2 & 169 & 13 & 24 & 2,4 & 312 & 312 & 1 & $\downarrow$ \\
\hline 52 & 52 & 1 & $\downarrow$ & 172 & 172 & 1 & $\downarrow$ & 313 & 313 & 96 & 10 \\
\hline 53 & 53 & 24 & 2 & 173 & 173 & 84 & 2 & 316 & 316 & 1 & $\downarrow$ \\
\hline 58 & 58 & 1 & $\downarrow$ & 178 & 178 & 1 & $\downarrow$ & 317 & 317 & 156 & 2 \\
\hline 59 & 59 & 28 & 2 & 179 & 179 & 88 & 2 & 330 & 330 & 1 & $\downarrow$ \\
\hline 60 & 60 & 1 & $\downarrow$ & 180 & 180 & 1 & $\downarrow$ & 331 & 331 & 80 & 3 \\
\hline 61 & 61 & 16 & 2 & 181 & 181 & 48 & 2 & 336 & 336 & 1 & $\downarrow$ \\
\hline 64 & 2 & 6 & $1,0,0,0,0,1$ & 190 & 190 & 1 & $\downarrow$ & 337 & 337 & 96 & 10 \\
\hline 66 & 66 & 1 & $\downarrow$ & 191 & 191 & 72 & 19 & 343 & 7 & 36 & $3,0,1$ \\
\hline 67 & 67 & 20 & 2 & 192 & 192 & 1 & $\downarrow$ & 346 & 346 & 1 & $\downarrow$ \\
\hline 70 & 70 & 1 & $\downarrow$ & 193 & 193 & 64 & 5 & 347 & 347 & 172 & 2 \\
\hline 71 & 71 & 24 & 7 & 196 & 196 & 1 & $\downarrow$ & 348 & 348 & 1 & $\downarrow$ \\
\hline 72 & 72 & 1 & $\downarrow$ & 197 & 197 & 84 & 2 & 349 & 349 & 112 & 2 \\
\hline 73 & 73 & 24 & 5 & 198 & 198 & 1 & $\downarrow$ & 352 & 352 & 1 & $\downarrow$ \\
\hline 78 & 78 & 1 & $\downarrow$ & 199 & 199 & 60 & 3 & 353 & 353 & 160 & 3 \\
\hline 79 & 79 & 24 & 3 & 210 & 210 & 1 & $\downarrow$ & 358 & 358 & 1 & $\downarrow$ \\
\hline 81 & 3 & 8 & $1,0,0,1$ & 211 & 211 & 48 & 2 & 359 & 359 & 178 & 7 \\
\hline 82 & 82 & 1 & $\downarrow$ & 222 & 222 & 1 & $\downarrow$ & 361 & 19 & 48 & 4,4 \\
\hline 83 & 83 & 40 & 2 & 223 & 223 & 72 & 3 & 366 & 366 & 1 & $\downarrow$ \\
\hline
\end{tabular}


Even with the new codes, there are still gaps in the available code lengths (Fig. 1). This raises the question of whether there could also be alternating codes for the missing lengths. We have done a complete search of codes with columns from the Fourier-matrices $F_{p}$ for all numbers up to $p=15$. For numbers followed by a prime there is indeed a unique code. On the other hand, for the numbers $8,9,14$ and 15 no alternating codes were found. These are the first composite numbers not followed by a prime. This small search hints to the possibility that there are no $p$-nary alternating codes of length $p$ (at least codes formed from $F_{p}$ ), except when $p$ or $p+1$ is a prime.

Acknowledgements. Support for J. Vierinen was provided by the Academy of Finland (application number 213476, Finnish Programme for Centres of Excellence in Research 2006-1011).

Topical Editor K. Kauristie thanks M. Sulzer and another anonymous referee for their help in evaluating this paper.

\section{References}

Lehtinen, M.: Statistical theory of incoherent scatter measurements, EISCAT Tech. Note 86/45, 1986.

Lehtinen, M. S., Huuskonen, A., and Markkanen, M.: Randomization of alternating codes: Improving incoherent scatter measurements by reducing correlations of gated ACF estimates, Radio Sci., 32, 2271-2282, 1997.

Lidl, R. and Niederreiter, H.: Finite Fields, 2nd ed., Cambridge University Press, 1997.

Markkanen, M. and Nygrén, T.: Long alternating codes: 2. Practical search method, Radio Sci., 32, 9-18, 1997.

Sulzer, M. P.: Recent incoherent scatter techniques, Adv. Space Res., 9, 153-162, 1989.

Sulzer, M. P.: A new type of alternating code for incoherent scatter measurements, Radio Sci., 28, 995-1001, 1993. 